\title{
The role of point-of-care blood testing for ketones in the diagnosis of diabetic ketoacidosis
}

\author{
A Coetzee, ${ }^{1}$ MMed (Int), FCP (SA); M Hoffmann, ${ }^{2}$ MMed (Chem Path); B H Ascott-Evans, ${ }^{1}$ FCP (SA) \\ ${ }^{1}$ Division of Endocrinology, Department of Medicine, Faculty of Medicine and Health Sciences, Stellenbosch University, Tygerberg, Cape Town, \\ South Africa \\ ${ }^{2}$ Department of Chemical Pathology, Tygerberg National Health Laboratory Service, Stellenbosch University, Tygerberg, Cape Town, South Africa
}

Corresponding author: A Coetzee (blommeland@gmail.com)

Background. Urine dipstick testing for ketones is widely used when diabetic ketoacidosis (DKA) is suspected in patients with hyperglycaemia. If urinary ketones are positive, patients are referred for further management - often inappropriately, as the test is a poor surrogate for plasma ketones. Plasma beta-hydroxybutyrate $(\beta-\mathrm{OHB})$ levels $>3 \mathrm{mmol} / \mathrm{L}$ are diagnostic of DKA, while levels $<1 \mathrm{mmol} / \mathrm{L}$ are insignificant.

Objectives. To evaluate a hand-held electrochemical (point-of-care testing; POCT) ketone monitor and compare it with the gold-standard manual enzymatic method (MEM) for detection of plasma ketones.

Methods. In a prospective and comparative study, we evaluated the measurement of $\beta$-OHB by means of POCT and the MEM in 61 consecutive samples from patients with suspected DKA at Tygerberg and Karl Bremer hospitals, Cape Town, South Africa. Capillary (for POCT) and plasma samples (for the MEM) were obtained simultaneously and compared for accuracy. Precision was assessed with control samples.

Results. The POCT method was precise (coefficient of variation $<4.5 \%$ ), and there was a good correlation between the two methods $(r=0.95)$. Regression analysis showed a proportional bias, with POCT reading higher than the MEM. However, when assessed at the relevant medical decision limits $(\beta-\mathrm{OHB}>3 \mathrm{mmol} / \mathrm{L}$ and $<1 \mathrm{mmol} / \mathrm{L}$ ), the total allowable error (bias + imprecision) was not exceeded. Patients will therefore still be classified correctly. The POCT method had a sensitivity of $100 \%$ and specificity of $89 \%$ for DKA $(\beta-O H B>3 \mathrm{mmol} / \mathrm{L})$, while at levels $<1 \mathrm{mmol} / \mathrm{L}$ sensitivity was $100 \%$ and specificity $87.5 \%$.

Conclusion. The POCT device provides an accurate and precise result and can be used as an alternative to the MEM in the diagnosis of DKA.

S Afr Med J 2015;105(9):756-759. DOI:10.7196/SAMJnew.7889

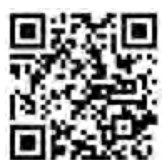

Diabetic ketoacidosis (DKA) is a common and severe acute complication of diabetes mellitus. The annual incidence is 46 - 50 episodes per 10000 diabetic patients. DKA accounts for $14 \%$ of hospital admissions of diabetic patients and $10 \%$ of all deaths from diabetes in the Western world. ${ }^{[1]}$ In sub-Saharan Africa the picture is bleaker, with mortality rates up to $30 \%{ }^{[2]}$ It has been estimated that the annual cost of treating DKA in the USA exceeds 1 billion $\mathrm{USD}^{[3]}$ and that the average cost per DKA episode is USD6 $500-7500$. This represented $25 \%$ of the total spent on the healthcare of patients with type 1 diabetes in $2010 .{ }^{[4]}$ No published costing data are available from South Africa (SA).

When significant hyperglycaemia occurs, it is important to know whether it is accompanied by ketosis or ketoacidosis. Highrisk patients should be identified early, so that timely measures can be taken to prevent the significant morbidity and mortality associated with DKA. Ketone measurement allows the clinician to distinguish accurately between simple hyperglycaemia and metabolic decompensation with ketoacidosis, thus speeding up referral and management of patients with suspected DKA, when appropriate.

Urine dipstick testing for ketones (Ketostix) is the screening test currently widely used in our drainage area when DKA is suspected in patients with hyperglycaemia. It is used as a 'surrogate' because serum ketone and blood gas $\mathrm{pH}$ measurements are not readily available in most state health centres. However, the dipstick method has limitations. The test is based on the nitroprusside reaction, which gives a semiquantitative measurement of acetoacetate. It reacts weakly with acetone, but does not measure beta-hydroxybutyrate $(\beta-\mathrm{OHB})$, and may give false-negative results. $\beta-\mathrm{OHB}$ is in fact the main metabolic product in the setting of DKA. It reflects the degree of ketosis, as levels of $\beta$-OHB correlate better than acetoacetate with falls in $\mathrm{pH}$ and bicarbonate levels. Early detection has been shown to lead to improved patient outcomes. ${ }^{[5]}$ Tests based on the nitroprusside reaction have also been reported to give false-positive results in various clinical scenarios such as the fasting state, pregnancy and the use of sulphydryl-containing medications, e.g. angiotensinconverting enzyme inhibitors. ${ }^{[6]}$ In a position statement, the American Diabetes Association (ADA) emphasises that urine ketone tests are not reliable for diagnosing DKA and that measurement of $\beta$-OHB in blood is preferred for diagnosis. ${ }^{[7]}$ At present, if urinary ketones are positive, these patients are referred to secondary or tertiary facilities for further management. This results in large numbers of patients being referred inappropriately as having DKA. Uncontrolled hyperglycaemia in the presence of a $\beta$-OHB level of $>3.0 \mathrm{mmol} / \mathrm{L}$ indicates unequivocal DKA, ${ }^{[8]}$ whereas a level of $<1 \mathrm{mmol} / \mathrm{L}$ excludes any significant ketosis. ${ }^{[9]}$

Quantitative $\beta$-OHB measurement is unfortunately not offered by the routine laboratory at Tygerberg Hospital (TBH), Cape Town, SA (a tertiary academic training hospital). Samples are referred across town to the Red Cross War Memorial Children's Hospital (RCWMCH) laboratory, and results are reported within 2 - 3 days. As a result, values cannot be used for decision-making in a medical emergency setting. Quantitative serum $\beta$-OHB measurement on a point-of-care testing (POCT) device has the potential to negate inappropriate referrals, which may translate into large cost savings if incorporated in initial patient assessment. It would also identify the 'false negatives' missed on Ketostix testing. POCT provides an attractive alternative to our current practice. Prior to its clinical use, it is essential that the POCT be evaluated to determine whether the device is accurate and precise and therefore fit for purpose. The 
Clinical and Laboratory Standards Institute (CLSI) has published guidelines to facilitate this evaluation process.

\section{Objectives}

(i) To evaluate a hand-held electrochemical (POCT) ketone monitor (Medisense/Abbott Optium Xceed) and compare it with the manual enzymatic method (MEM), the current gold standard, to assess its accuracy and precision; (ii) to assess diagnostic performance of the POCT v. the MEM; and (iii) to calculate possible cost implications of utilising POCT as an alternative to the MEM.

\section{Methods \\ Study design and setting}

The study was prospective and comparative, evaluating the measurement of $\beta-\mathrm{OHB}$ by means of electrochemical POCT and the MEM in consecutive patients referred from primary levels of care with suspected DKA. Referral was based on hyperglycaemia (capillary glucose $>13.9 \mathrm{mmol} / \mathrm{L}$ ) and the presence of urinary ketones on dipstick testing.

The study was conducted over a 4-month period in the acute medical admission ward and the high-care ward at $\mathrm{TBH}$ as well as in the acute medical admission ward at Karl Bremer Hospital (KBH), also in Cape Town. Blood samples were collected at admission and during treatment to ensure that a wide range of ketone values would be obtained. Patients were assessed clinically and attempts were made to identify the precipitant of the DKA episode.

\section{Analytical performance}

Method validations are performed to assess the degree of error inherent in a method and to determine whether the inaccuracy/bias and imprecision of the method will affect the interpretation of a test. CLSI documents EP09[10] ("Method comparison and bias estimation using patient samples') and EP15 ${ }^{[11]}$ ('User verification of performance for precision and trueness') (www.clsi.org) were followed. These documents state that precision can be determined using quality control samples, and that accuracy can be assessed by analysing at least 40 samples spanning the measurement range.

POCT was performed using the Medisense/Abbott Optium Xceed $\beta-\mathrm{OHB}$ meter, and results were expressed in $\mathrm{mmol} / \mathrm{L}$. Laboratory testing was performed by means of a MEM, results being expressed in $\mu \mathrm{mol} / \mathrm{L}$ and then converted to $\mathrm{mmol} / \mathrm{L}$. The investigators were blinded to the results.

For precision studies, quality control samples at two levels close to the medical decision limits were provided by the manufacturer. Samples were analysed in triplicate over a 5-day period on the POCT device. The mean, standard deviation (SD) and coefficient of variation (CV, \%) (mean/ $\mathrm{SD} \times 100)$ were calculated and compared with the manufacturer's published results as per the package insert. The manufacturer claims a total imprecision of $8.8 \%$ at a $\beta-\mathrm{OHB}$ level of $0.7 \mathrm{mmol} / \mathrm{L}$ and a total imprecision of $3.1 \%$ at a $\beta$-OHB level of $4.3 \mathrm{mmol} / \mathrm{L}^{\text {[12] }}$

Accuracy studies were performed on 61 samples collected from the 41 patients enrolled in the study. Samples from consecutively presenting patients were used with ketone levels that spanned the measurement range and included clinical decision-making levels. Capillary and serum samples were obtained at the same time. Capillary blood was used for POCT. Venous whole blood was collected in lithium heparin tubes (BD Vacutainer) and immediately transported on ice to the TBH National Health Laboratory Service (NHLS) laboratory. The transit time ranged from 10 to 20 minutes. At the laboratory, samples were immediately deproteinised with perchloric acid as per the RCWMCH protocol, stored at $-70^{\circ} \mathrm{C}$ and transported to RCWMCH within 48 hours. At the RCWMCH laboratory the samples were stored at $-20^{\circ} \mathrm{C}$ and analysed between 1 and 7 days of arrival. According to the literature, plasma samples are stable for several weeks at $-20^{\circ} \mathrm{C} .^{[13]}$

Linear regression analysis and a BlandAltman plot were used for analytical comparison and for depicting allowable bias and total allowable error. Method comparison statistics was performed with the Analyse-It version 2.3 data package for Excel.

\section{Diagnostic performance}

Sensitivity and specificity were calculated at the clinical decision-making limits ( $1 \mathrm{mmol} / \mathrm{L}$ and $3 \mathrm{mmol} / \mathrm{L}$ ) to assess diagnostic performance.

\section{Cost}

The cost of reagent strips for POCT was borne by the manufacturer. The cost of the MEM was borne by the NHLS.

The cost for POCT is currently ZAR22 per strip, and the POCT device costs ZAR249. The cost of MEM for detection of serum ketones (including $\beta-\mathrm{OHB}+$ acetoacetate) is ZAR228.88.

\section{Results}

\section{Demographics and descriptive parameters}

Most patients seen at $\mathrm{TBH}$ and $\mathrm{KBH}$ are of mixed ancestry, the gender and ethnic distribution being representative of the general population served by these hospitals. In total, 61 samples were collected from 41 patients, of whom 24 were females and 17 males. The mean age was 33 years (range 17 $52)$. The majority had type 1 diabetes $(n=29$, $70.7 \%$ ), seven being newly diagnosed. All the patients were found to have ketones present on dipstick testing of the urine, ranging from $1+$ to $3+$. Of the 41 referred patients with suspected DKA, only 15 were true DKA cases when using MEM ketones ( $>3 \mathrm{mmol} / \mathrm{L}$ ) and hyperglycaemia (fingerprick blood glucose $>13.9 \mathrm{mmol} / \mathrm{L}$ ). The majority of the patients $(n=26,63.4 \%)$ were therefore inappropriately referred on the basis of positive urinary

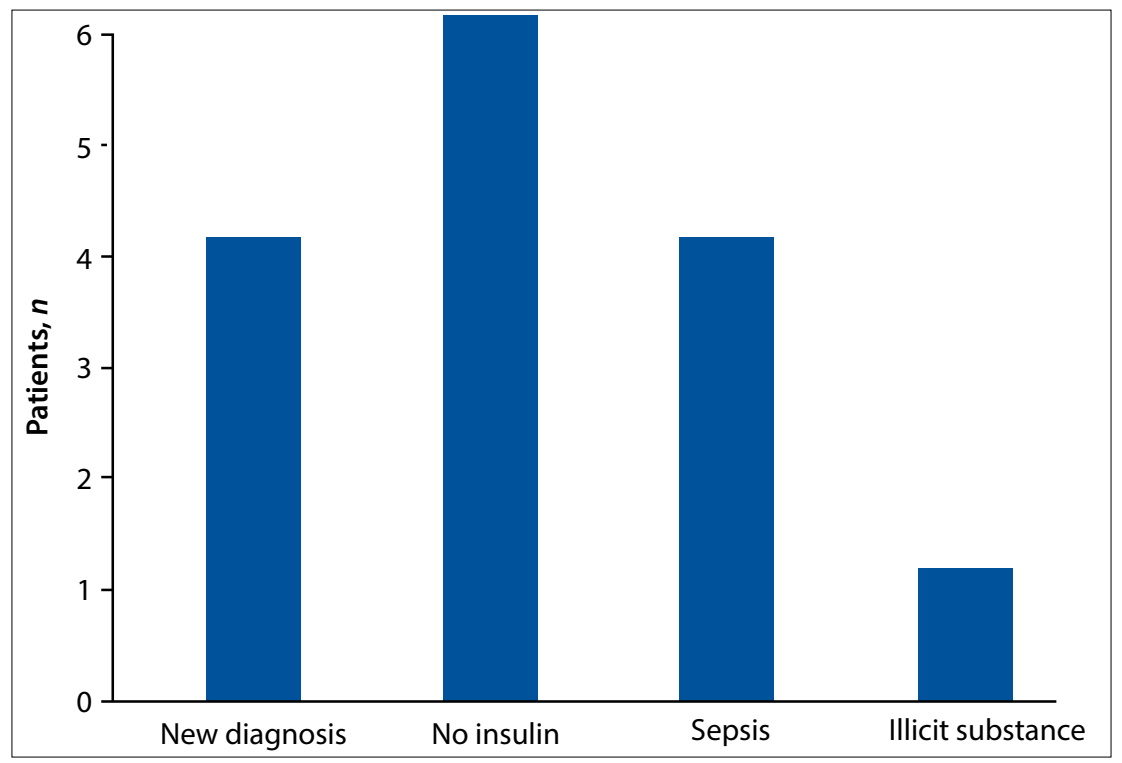

Fig. 1. Precipitants of DKA. 
ketones and hyperglycaemia. When evaluating ketosis $(\beta-\mathrm{OHB}$ $>1 \mathrm{mmol} / \mathrm{L}$ and $<3 \mathrm{mmol} / \mathrm{L}$ ), an additional five patients had ketosis but not ketoacidosis. Even if these five patients are added to the 15 with DKA, more than half of all the patients $(n=21,51.2 \%)$ were inappropriately referred.

The factors that precipitated the DKA episodes are shown in Fig. 1. Although our study was not designed to investigate the causes thereof, it was clear that lack of adherence to insulin therapy and sepsis seemed to be the major contributors.

\section{Analytical performance}

\section{Precision}

Precision of POCT was assessed by adhering to guidelines of the CLSI. ${ }^{[14]} \mathrm{A}$ summary of the results is presented in Table 1. At both low and high control levels, the total imprecision of the POCT device did not exceed the imprecision claimed by the manufacturer. Manufacturer claims were therefore verified at both control levels.

\section{Accuracy}

As the data were not normally distributed, non-parametric statistics were used. Spearman rank order correlation, performed with regard to actual values in $\mathrm{mmol} / \mathrm{L}$ of both results obtained from POCT and the MEM (Fig. 2), demonstrated a very high correlation between the two methods $(r=0.95)$. Since high correlation coefficients do not imply agreement of methods, we examined the agreement using the Bland-Altman difference plot and depicting allowable bias $(18.4 \%)$ and total allowable error $(68.7 \%) \cdot{ }^{[15]}$ The Bland-Altman plot demonstrated a proportional bias, with the POCT device reading higher than the MEM. The bias exceeded the total allowable bias, but when combined with the precision, did not exceed the total allowable error (Fig. 3). ${ }^{[16]}$ When assessed at medical decision limits, the positive bias was $0.22 \mathrm{mmol} / \mathrm{L}$ at $1 \mathrm{mmol} / \mathrm{L}(p=0.77)$ and 0.68 $\mathrm{mmol} / \mathrm{L}$ at $3 \mathrm{mmol} / \mathrm{L}(p=0.85)$.

\section{Diagnostic performance}

When compared with the MEM, the POCT device demonstrated a sensitivity of $100 \%$ and a specificity of $89.5 \%$ for diagnosing DKA $(\beta-\mathrm{OHB}>3 \mathrm{mmol} / \mathrm{L})$ and a sensitivity of $100 \%$ and specificity of $87.5 \%$ for excluding DKA $(\beta-\mathrm{OHB}<1 \mathrm{mmol} / \mathrm{L})$ (Table 2$)$. Other performance criteria are summarised in Table 2 .

\section{Discussion}

DKA is a common complication in our diabetic population. It is clear that the majority of patients were inappropriately referred with suspected DKA, and that this might have been avoided had the means to detect serum ketones been available at referral centres.

Only a minority of patients had $\mathrm{pH}$ measurements before being referred. Is it then correct to diagnose DKA at a certain level of $\beta$-OHB (in an uncontrolled diabetic), without knowing the $\mathrm{pH}$ level? Ketone body anion concentrations directly reflect the rate of ketone body production, which is accompanied by equimolar production of

Table 1. Manufacturer claims for precision of the POCT device validated using control samples

\begin{tabular}{llll}
\hline $\boldsymbol{\beta}$-OHB, & & & \\
mmol/L & Claimed CV, \% & Obtained CV, \% & Claim verified \\
\hline 0.7 & 8.8 & 4.3 & Yes \\
4.3 & 3.1 & 2.3 & Yes
\end{tabular}

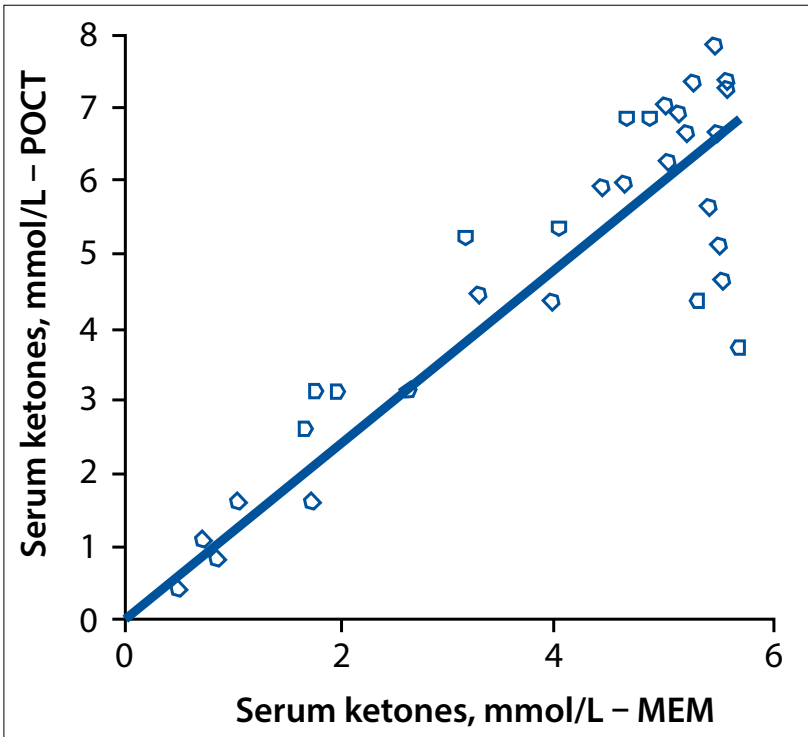

Fig. 2. Scatter plot showing correlation between the POCT method and the MEM.

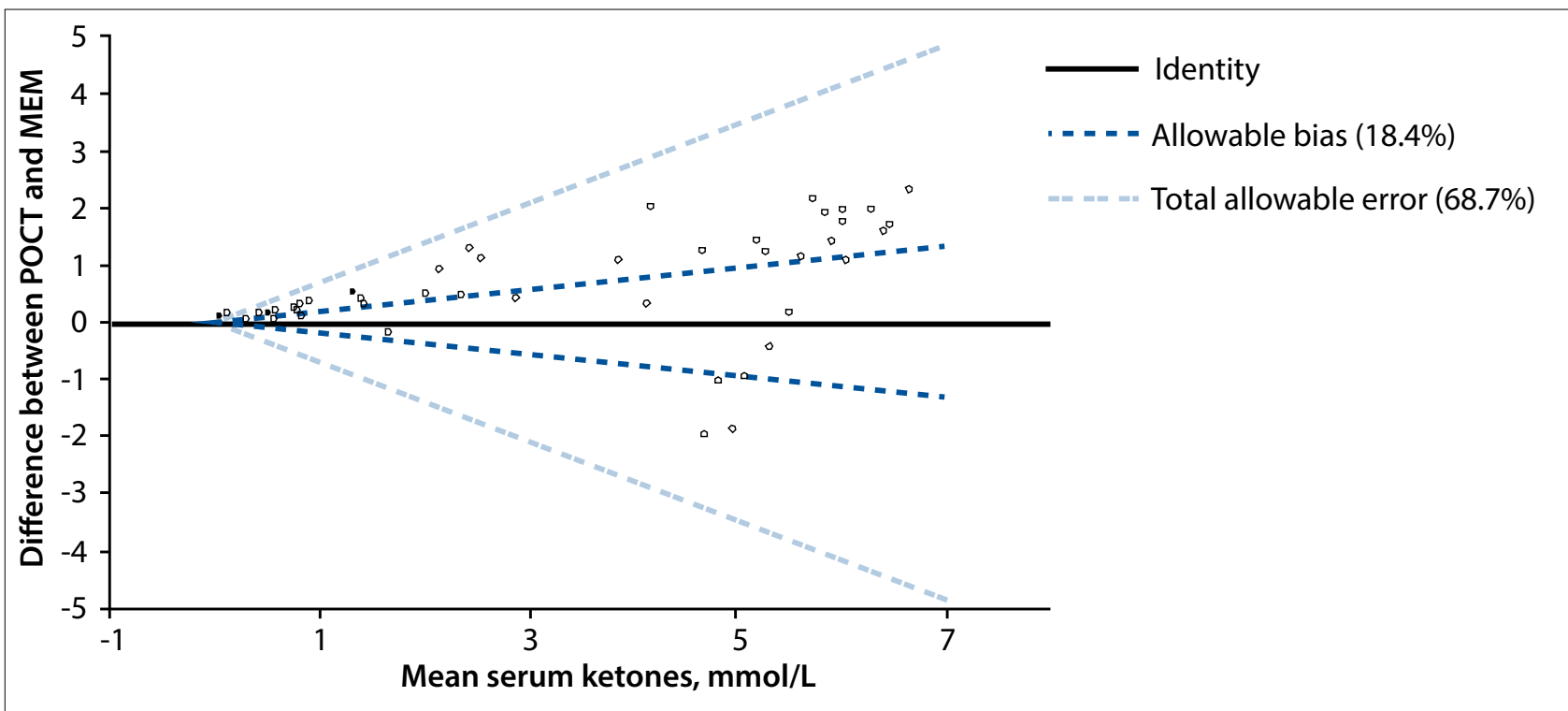

Fig. 3. Bland-Altman plot depicting allowable bias and total allowable error. 
Table 2. Ability of the POCT device to diagnose DKA and ketosis

\begin{tabular}{ll}
\hline & $n / N(\%)$ \\
\hline DKA $(\beta-\mathrm{OHB}>3 \mathrm{mmol} / \mathrm{L})$ & \\
Sensitivity & $23 / 23(100.0)$ \\
Specificity & $34 / 38(89.5)$ \\
Positive predictive value & $23 / 27(85.2)$ \\
Negative predictive value & $34 / 34(100.0)$ \\
Ketosis excluded $(\beta-\mathrm{OHB}<1 \mathrm{mmol} / \mathrm{L})$ & \\
Sensitivity & $33 / 33(100.0)$ \\
Specificity & $28 / 32(87.5)$ \\
Positive predictive value & $33 / 37(89.2)$ \\
Negative predictive value & $28 / 28(100.0)$
\end{tabular}

hydrogen ions. Over the years numerous proposed markers and cutoffs have confirmed that any diagnostic criterion for DKA is arbitrary. This led the ADA to issue a consensus statement in 2006 that included set levels of $\mathrm{pH}, \mathrm{HCO}_{3}$, ketones, anion gap and glucose. ${ }^{[17]}$ While all five of these criteria have serious limitations, the most robust criterion is regarded to be a serum bicarbonate level of $<18 \mathrm{mEq} / \mathrm{L}$. However, in a 2008 Mayo Clinic study comparing this cut-off level with appropriate levels of $\beta-\mathrm{OHB}$, there was a statistically strong correlation overall, but $16 \%$ false-positive and $17 \%$ false-negative rates at this bicarbonate level. Similarly, when a pH of $<7.3$ was the comparator, $20 \%$ of DKA patients had a false-negative $\mathrm{pH}^{\left[{ }^{[8]}\right.}$

Although our study was not designed to investigate the causes of DKA, it was clear that lack of adherence to insulin therapy and sepsis seemed to be the major contributors. All the patients we evaluated were positive for urinary ketones on dipstick testing at the referring centres.

Measurement of urinary ketones has various limitations, especially in the diagnosis of DKA. Reliable quantitative serum ketone measurement is preferable. Measurement of serum ketones has many applications in the management of patients with diabetes. The need for a rapid, reliable and accurate method led to the development of POCT for the measurement of $\beta$-OHB. In our study we evaluated a handheld electrochemical POCT ketone monitor v. the MEM (current gold standard) and evaluated its use in a resource-limited setting.

The method validation performed in this study proved that POCT was precise and accurate. The POCT device did show a proportional bias (increase in the magnitude of the error as the test result increased), which exceeded the allowable bias as stated in the literature. However, when taking the low imprecision of the method into account, the total error did not exceed the total allowable error (bias + imprecision), making this method acceptable. The performance was also evaluated at the medical decision limits of $1 \mathrm{mmol} / \mathrm{L}$ (rule out DKA) and $3 \mathrm{mmol} / \mathrm{L}$ (rule in DKA). This confirmed that patients will not be incorrectly classified because of the observed bias. The POCT device was therefore deemed fit for purpose.

How should diabetic patients with $\beta$-OHB in the ketosis range be managed? Five of our patients had levels between 1 and $3 \mathrm{mmol} / \mathrm{L}$ with the MEM, and all five were also within this range on POCT. In general, it should therefore not be necessary to up-refer these patients. However, if such patients look unexpectedly ill or acidotic, they should be referred, as other factors such as lactic acidosis or renal failure may be present.
The current cost to user of performing a serum ketone test is ZAR228.88. This includes a serum qualitative ketone determination as well as acetoacetate and $\beta$-OHB quantitative determination. The cost of the POCT is ZAR22 per strip. For this study the point of care machine (Abbott OptiumXceed) was provided free of charge by the manufacturer. It retails for ZAR205 - 249. Even adding capital outlay, cost of disposables and sundries, it is clear that POCT is far more cost-effective than the MEM.

\section{Study limitations}

The aim of the study was to evaluate POCT in measuring blood ketones and not to ratify POCT for treatment monitoring. POCT was not incorporated in the decision-making in this study, so we cannot comment on its use to improve outcomes. Although use of POCT appears to be more economical than the MEM, a formal cost analysis was not performed. Consecutive patient samples were used for the method comparison, so the spread of results advised by the CLSI documents/Westgard Basic Method Validation guidelines ${ }^{[14]}$ (which state that one-third of results must be in the low to low-normal range, one-third in the normal range, and one-third in the high-abnormal range) could not be followed.

\section{Conclusions}

The Abbot OptiumXceed POCT device provides an accurate and precise blood $\beta$-OHB result. This device offers a cost-effective practical alternative to laboratory measurement of ketones in patients with suspected DKA. Its adoption would bypass the use of urine dipstick testing to diagnose DKA, leading to more appropriate referrals and major cost savings. Our findings may potentially lead to better management of patients with DKA. However, appropriately designed studies will be needed to confirm this.

\section{References}

1. Faich GA, Fishbein HA, Ellis SE. The epidemiology of diabetic acidosis: A population-based study. Am J Epidemiol 1983;117(5):551-558.

2. Otieno CF, Kayima JK, Omonge EO, Oyoo GO. Diabetic ketoacidosis: Risk factors, mechanisms and management strategies in sub-Saharan Africa: A review. East Afr Med J 2005;82(12 Suppl):S197-S203.

3. Javor KA, Kotsanos JG, McDonald RC, Baron AD, Kesterson JG, Tierney WM. Diabetic ketoacidosis charges relative to medical charges of adult patients with type 1 diabetes. Diabetes Care 1997;20(3):349354. [http://dx.doi.org/10.2337/diacare.20.3.349]

4. Maldonado MR, Chong ER, Oehl MA, Balasubramanyam A. Economic impact of diabetic ketoacidosis in a multiethnic indigent population. Diabetes Care 2003;26(4):1265-1269. [http://dx.doi.org/10.2337/ diacare.26.4.1265]

5. Vanelli M, Chiari G, Capuano C. Cost effectiveness of the direct measurement of 3-betahydroxybutyrate in the management of diabetic ketoacidosis in children. Diabetes Care 2003;26(3):959. [http://dx.doi.org/10.2337/diacare.26.3.959]

6. Csako G. False-positive results for ketone with the drug mesna and other free-sulfhydryl compounds. Clin Chem 1987;33(2):289-292.

7. Goldstein DE. Tests of glycemia in diabetes. Diabetes Care 2004;27(Suppl 1):S91-S93. [http://dx.doi. org/10.2337/diacare.27.7.1761]

org/10.2337/diacare.27.7.1761]
8. Sheikh-Ali M, Karon BS, Basu A, et al. Can serum beta-hydroxybutyrate be used to diagnose diabetic Sheikh-Ali M, Karon BS, Basu A, et al. Can serum beta-hydroxybutyrate be used to diagn
ketoacidosis? Diabetes Care 2008;31(4):643-647. [http://dx.doi.org/10.2337/dc07-1683]

ketoacidosis? Diabetes Care 2008;31(4):643-647. [http://dx.doi.org/10.2337/dc07-1683]
9allace TM, Matthews DR. Recent advances in the monitoring and management of diabetic 9. Wallace TM, Matthews DR. Recent advances in the monitoring and manage
ketoacidosis. QJM 2004;97(12):773-780. [http://dx.doi.org/10.1093/qjmed/hch132]

10. Clinical and Laboratory Standards Institute. Method comparison and bias estimation using patient samples. EP09-A2, Vol. 13, No. 17:1-80.

11. Clinical and Laboratory Standards Institute. User verification of performance for precision and trueness. EP15-A2, Vol. 25, No. 17:1-64.

12. Abbott Diabetes Care Inc. Clinical evaluation of a faster, smaller sample volume blood beta ketone test strip. http://www.abbottdiabetescare.co.uk/_resources/media/documents/hcps/clinical_papers/08_ KII_white\%20_paper.pdf (accessed 1 October 2014).

13. Sacks DB, Arnold M, Bakris GL, et al. Guidelines and recommendations for laboratory analysis in the diagnosis and management of diabetes mellitus. Diabetes Care 2011;34(6):e61-e99. [http://dx.doi. org/10.2337/dc11-9998, http://dx.doi.org/10.2337/dc11-9997]

14. Chesher D. Evaluating assay precision. Clin Biochem Rev 2008;29(Suppl. 1):S23-S26.

14. Chesher D. Evaluating assay precision. Clin Biochem Rev 2008;29(Suppl. 1):S23-S26.
15. Widjaja A, Morris RJ, Levy JC, Frayn KN, Manley SE, Turner RC. Within and between subject variation in commonly measured anthropometric and biochemical variables. Clin Chem 1999;45(4):561-566. in commonly measured anthropometric and biochemical variables. Clin Chem 1999;45(4):
6. Westgard JO. Basic Method Validation. 3rd ed. Madison, WI: Westgard QC, 2008:27-84.

16. Westgard JO. Basic Method Validation. 3rd ed. Madison, WI: Westgard QC, 2008:27-84.
17. Kitabchi AE, Umpierrez GE, Murphy MB, Kreisberg RA. Hyperglycemic crises in adult patients with diabetes: A consensus statement from the American Diabetes Association. Diabetes Care 2006;29(12):2739-2748. [http://dx.doi.org/10.2337/dc06-9916]

Accepted 4 July 2015 